\title{
Alterations in the morphology of dendrites and dendritic spines in the nucleus accumbens and prefrontal cortex following repeated treatment with amphetamine or cocaine
}

\author{
Terry E. Robinson and Bryan Kolb ${ }^{1}$ \\ Department of Psychology and Neuroscience Program, The University of Michigan, 525 E. University, Ann Arbor, \\ MI 48109, USA \\ ${ }^{1}$ Department of Psychology, University of Lethbridge, Lethbridge, Alberta, Canada T1K 3M4
}

Keywords: Golgi, morphology, neuron structure, neuroplasticity, psychostimulant drugs, sensitization

\begin{abstract}
Repeated treatment with psychostimulant drugs produces changes in brain and behaviour that far outlast their initial neuropharmacological actions. The nature of persistent drug-induced neurobehavioural adaptations is of interest because they are thought to contribute to the development of dependence and addiction, and other forms of psychopathology, e.g. amphetamine psychosis. There are many reports that psychostimulants produce biochemical adaptations in brain monoamine systems, especially dopamine systems. The purpose of the present study was to determine if they might also alter the morphology of neurons in brain regions that receive monoaminergic innervation. Rats were given repeated injections of either amphetamine or cocaine, or, to control for general motor activity, allowed access to a running wheel. They were then left undisturbed for 24-25 days before their brains were processed for Golgi-Cox staining. Treatment with either amphetamine or cocaine (but not wheel running experience) increased the number of dendritic branches and the density of dendritic spines on medium spiny neurons in the shell of the nucleus accumbens, and on apical dendrites of layer $\mathrm{V}$ pyramidal cells in the prefrontal cortex. Cocaine also increased dendritic branching and spine density on the basilar dendrites of pyramidal cells. In addition, both drugs doubled the incidence of branched spines on medium spiny neurons. It is suggested that some of the persistent neurobehavioural consequences of repeated exposure to psychostimulant drugs may be due to their ability to reorganize patterns of synaptic connectivity in the nucleus accumbens and prefrontal cortex.
\end{abstract}

\section{Introduction}

The repeated administration of potentially addictive drugs results in many long-lasting changes in brain and behaviour. For example, some drug effects show tolerance while at the same time others show sensitization, and these decreases or increases in drug effect may persist long after the discontinuation of drug treatment (Robinson \& Becker, 1986; Kalivas \& Stewart, 1991; Stewart \& Badiani, 1993). Similarly, environmental stimuli associated with drug administration may acquire potent conditioned stimulus properties, and thus the ability to modify ongoing behaviour even in the absence of the drug (Davis \& Smith, 1976; Tomie, 1996). How experience with drugs of abuse changes the brain to produce persistent changes in behaviour is of great interest because of the potential role of drug-induced neurobehavioural adaptations in the development of dependence and addiction, and in craving and relapse (Nestler et al., 1993; Robinson \& Berridge, 1993).

Most research on neuroadaptations induced by psychostimulant drugs has focussed on biochemical adaptations in brain monoamine systems (Robinson \& Becker, 1986; Kalivas \& Stewart, 1991; Stewart

Correspondence: T.E. Robinson, as above. E-mail: ter@umich.edu

Received 9 September 1998, revised 24 November 1998, accepted 16 December 1998
\& Badiani, 1993), and more recently, in excitatory amino acid neurotransmission (Pierce \& Kalivas, 1997; Wolf, 1998). This is because the primary neuropharmacological effect of amphetamine and cocaine is on monoamine transporters, where they act to increase the extracellular concentrations of dopamine, norepinephrine and serotonin (Kuczenski, 1983). Amphetamine and cocaine produce a host of biochemical adaptations in monoamine systems, and excitatory amino acid neurotransmission, many of which could contribute to their persistent effects on behaviour (Robinson \& Becker, 1986; Kalivas \& Stewart, 1991; White \& Wolf, 1991; Stewart \& Badiani, 1993; Pierce \& Kalivas, 1997; Wolf, 1998). However, stimuli or events that produce very persistent changes in behaviour typically alter not only brain biochemistry, but also brain structure, modifying patterns of synaptic connectivity (Greenough et al., 1990; Kolb et al., 1998). Given that psychostimulant drugs have extremely potent stimulus properties, and that they can produce persistent changes in behaviour, it seems reasonable to hypothesize that they might also alter patterns of synaptic connectivity. Consistent with this hypothesis, we have found that treatment with escalating doses of amphetamine $(1-10 \mathrm{mg} / \mathrm{kg}$ ) alters the structure of neuronal dendrites in the nucleus accumbens and prefrontal cortex (Robinson \& Kolb, 1997).

The purpose of the present experiment was threefold. First, we sought to determine whether a less aggressive amphetamine treatment regimen, more typical of those used in studies of amphetamine 
sensitization (Robinson \& Becker, 1986), would also alter the morphology of neurons in the nucleus accumbens and prefrontal cortex. Second, we sought to determine whether repeated treatment with cocaine, another widely abused psychostimulant drug, would produce comparable effects. Third, we included a 'motor activity' control group to determine whether the effects of amphetamine and/ or cocaine might simply be secondary to their ability to increase motor activity. To address these questions, rats were treated with amphetamine or cocaine (or to control for general motor activity allowed access to a running wheel), and 1 month later their brains were processed for Golgi-Cox staining (Kolb et al., 1998). This method is sensitive to experience-dependent changes in neuronal morphology (Greenough et al., 1990; Kolb et al., 1998), and allowed us to quantify the effects of drug treatment on dendritic length, spine density and the incidence of branched spines on medium spiny neurons in the shell of the nucleus accumbens and layer $\mathrm{V}$ pyramidal cells in the medial prefrontal cortex.

\section{Methods}

Female Sprague-Dawley rats weighing 200-225 g at the beginning of the experiment were housed singly in wire-hanging cages in a room on a 14:10 h light:dark cycle, with food and water available $a d$ libitum. After 1 week, the rats were randomly assigned to one of four groups. Three groups received an intraperitoneal injection once each day for five consecutive days, followed by two injection-free days, and this procedure was repeated for four consecutive weeks. The animals received injections of either: $0.9 \%$ saline, $3 \mathrm{mg} / \mathrm{kg}$ D-amphetamine sulphate or $15 \mathrm{mg} / \mathrm{kg}$ of cocaine $\mathrm{HCl}$ (drug doses weight of the salt). The animals in the saline-treated control group were also used in a parallel unpublished study with morphine. All injections were given in activity monitors described previously (Camp \& Robinson, 1988). On the day of the first injection, animals in the fourth group were housed in cages attached to running wheels, to which they had free access $24 \mathrm{~h} /$ day. They were left in the running wheels for 4 weeks, and were then returned to the standard wirehanging cages. This latter group was included as a motor activity control because female rats allowed access to running wheels show very high levels of motor activity. To the extent that any changes in brain structure produced by amphetamine or cocaine are simply a consequence of the increase in motor activity produced by these agents, then animals that engage in high levels of non-drug-dependent motor activity should show comparable effects.

After 4 weeks of injections or running wheel experience, all animals were left undisturbed for 24-25 days. They were then deeply anaesthetized with sodium pentobarbital and perfused intracardially with $0.9 \%$ saline. The brains were removed and placed in vials containing Golgi-Cox solution, and after 14 days transferred to vials containing $30 \%$ sucrose. After at least 3 days in the sucrose solution, the brains were cut into $200 \mu \mathrm{m}$ coronal sections using a vibratome, and stained using procedures described elsewhere (Gibb $\&$ Kolb, 1998). The modified Golgi-Cox procedure used here is less capricious than traditional methods and allows for the consistent visualization of dendritic spines (Gibb \& Kolb, 1998; Kolb et al., 1998).

Cells in two brain regions were selected for analysis: medium spiny neurons in the shell of the nucleus accumbens and layer $\mathrm{V}$ pyramidal neurons in the medial prefrontal cortex (area $\mathrm{Cg} 3$, according to Zilles, 1985). These cells were selected for study because: (i) these regions are thought to contribute to the psychomotor activating and rewarding effects of amphetamine and cocaine; (ii) these neurons represent the major output neurons in these structures; and (iii) in a previous study we found that amphetamine altered the morphology of neurons in these regions (Robinson \& Kolb, 1997). The brain regions were first identified at low power $(100 \times)$, and five cells from each hemisphere were drawn using camera lucida (at $250 \times$ ). In order to be included in the analysis, the dendritic tree of a cell could not be obscured with stain precipitations, blood vessels or astrocytes, and the dendritic fields had to be well impregnated and appear largely intact and visible in the plane of section. The dendritic surface was quantified using two methods. First, the total number of dendritic branches (indicated by bifurcations) was counted at each order away from the cell body or dendritic shaft (Coleman \& Riesen, 1968). Second, dendritic length was estimated by counting the number of ring intersections using an overlay of concentric rings (Sholl, 1981). To calculate spine density, a length of dendrite (at least $>10 \mu \mathrm{m}$ long) was traced (at $1000 \times$ ), the exact length of the dendritic segment was calculated, and the number of spines along that length counted (to yield spines $/ 10 \mu \mathrm{m}$ ). For pyramidal cells, spines were counted on one third-order tip from both the basilar and apical dendrites, and for medium spiny neurons spines on one terminal tip were counted.

In a second analysis of only medium spiny neurons in only control and drug-treated animals, the frequency of branched spines was determined by drawing dendritic segments at $2000 \times$, and counting both total spines and spines with more than one head. The dendritic segment analysed in this instance was located on the opposite side of the cell from the one initially analysed. Branched spines were defined as spines with more than one head clearly connected to a common shaft, as described by Comery et al. (1996). Thus, this analysis represented both a partial replication of the initial analysis because different dendritic segments were drawn, and an extension to determine if amphetamine or cocaine had a disproportionate effect on the number of branched spines (i.e. those with multiple heads).

The person responsible for cell selection and drawing was blind to the experimental conditions. Statistical analyses were performed by averaging across cells per hemisphere; i.e. hemisphere was the unit of analysis. The value of $N$ was 12 for the control group and 10 for each of the experimental groups. Group differences were determined by one-way ANOVAs followed by Scheffe comparisons (with $\alpha=0.05$ for Scheffe tests). For those groups in which there was a significant overall effect on the total number of branches, the effect of branch order was determined using one-way ANOVAs at each branch order, and if indicated, Bonferroni-Dunn corrected $t$-tests to compare each experimental group with the control group.

\section{Results}

\section{Behaviour}

As has been reported many times, both amphetamine and cocaine produced an increase in locomotor activity, and sensitization was evident with repeated treatment (Fig. 1). For amphetamine there was a significant increase in cage crossovers between the first and last (20th) test session $[t(9)=2.57, P=0.030]$. Similarly, there was a greater locomotor response to cocaine on the last test session than on the first $[t(7)=5.81, P<0.001]$. In contrast, the response to a saline injection decreased between the first and last test session $[t(8)=P=0.016$ ), presumably due to habituation to the test environment.

As predicted, animals housed in running wheels showed high levels of motor activity. On average, over the entire time they were in the wheels, these rats made $4862 \pm 882$ revolutions $/ 24 \mathrm{~h}$. Given the circumference of the wheel, this represents a distance of approxi- 


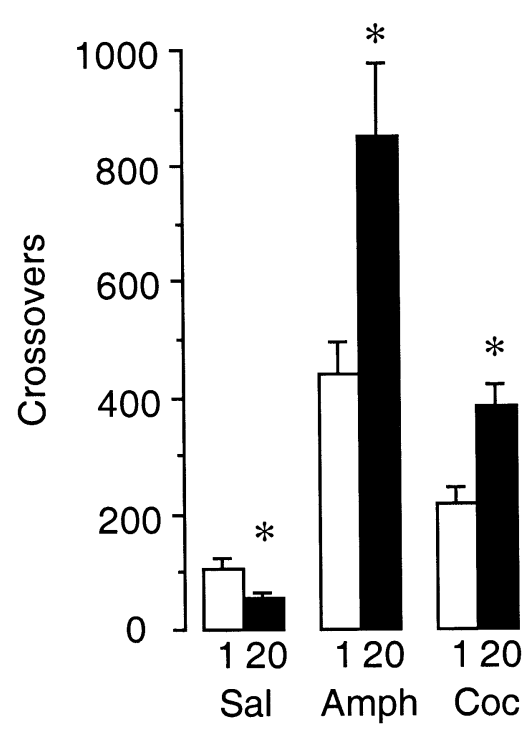

FIG. 1. Mean ( \pm SEM) number of cage crossovers averaged over the first (open bars) and 20th (closed bars) test session in rats given repeated injections of saline (Sal), amphetamine (Amph) or cocaine (Coc). Asterisks indicate that the response on day 20 differed significantly from the response on day 1 (paired $t$-tests)

mately $5.43 \mathrm{~km} / 24 \mathrm{~h}$. For comparison, a rough calculation of the distance travelled (cage crossovers) during an average test session with cocaine yields a value of $0.14 \mathrm{~km} / 1.5 \mathrm{~h}$ test session.

\section{Anatomy}

There was an effect of drug treatment on the structure of dendrites in both brain regions examined. Figure 2A shows that in the shell of the nucleus accumbens there was a $6.9 \%$ increase in the total number of dendritic branches in both amphetamine- and cocaine-treated rats, relative to controls, and a 5.3\% decrease in total dendritic branches in rats housed in running wheels (overall ANOvA, $F=20.9, P<0.001$, follow-up Scheffe tests, $P<0.05$ ). To determine which portion of the dendritic tree was affected by each manipulation, the number of branches at each order from the cell body was also analysed. The decrease in the number of branches seen in the wheel group was statistically significant only for second- and third-order branches (Fig. 3). In contrast, the psychostimulants altered more distal branches. In amphetamine-treated animals, there was a significant increase only in the number of fifth-order branches. In cocaine-treated rats, there was a significant increase in both fifth- and sixth + -order branches (Fig. 3).

Drug treatment also significantly increased the density of spines on medium spiny neurons $(F=117.2, P<0.001$; Fig. 2D). In the amphetamine-treated group, spine density was increased by $11.2 \%$ and in the cocaine group by $13.5 \%$. There was no effect of wheel running experience on spine density (Fig. 2D). Figure 4 shows camera lucida drawings of representative medium spiny neurons and dendritic segments from control and drug-treated animals.

Figure 5 shows the results of the second analysis of medium spiny neurons to examine the incidence of branched spines. As in the first analysis, there was a significant increase in the total number of spines in both amphetamine- $(12.3 \%$ increase relative to control) and cocaine(17.2\% increase) treated animals $(F=136.0, P<0.001$; Fig. 5A). Figure 5B shows that there was a disproportionate increase in the number of spines with multiple heads $(F=156.0, P<0.001)$. The number of branched spines was increased by $197.7 \%$ in amphetaminetreated rats and $132.6 \%$ in cocaine-treated rats. Thus, in control

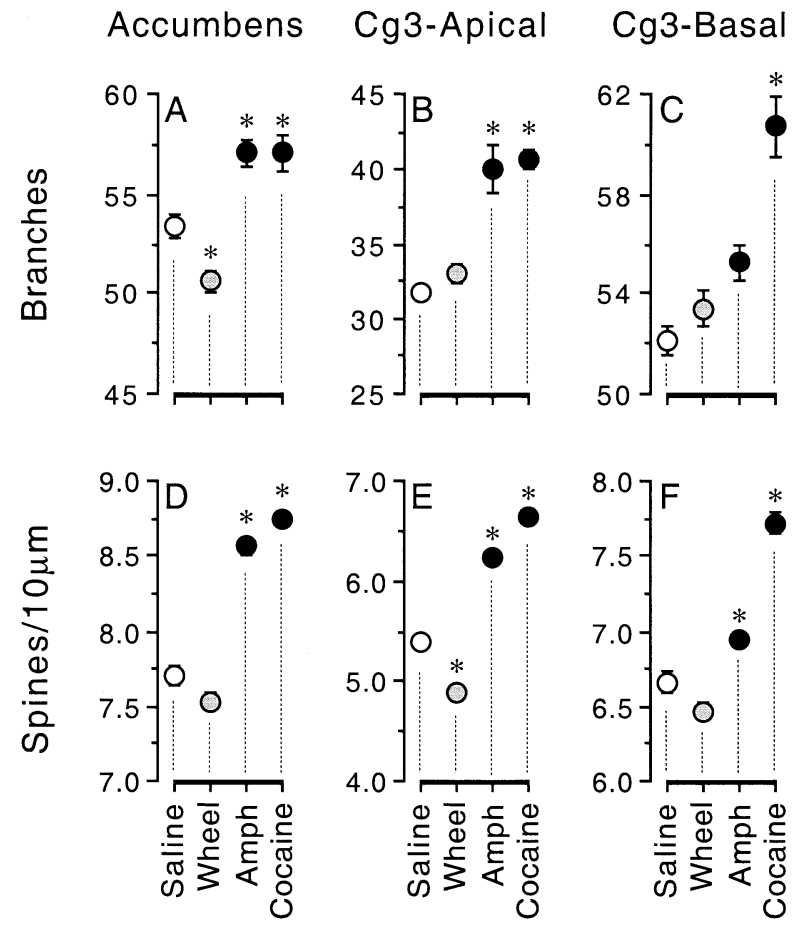

FIG. 2. Quantitative analysis of the total number of dendritic branches (top) and spine density (bottom) on medium spiny neurons in the shell of the nucleus accumbens (A,D) and on apical (Cg3-Apical, B,E) and basilar (Cg3Basal, C,F) dendrites of layer $\mathrm{V}$ pyramidal cells in the medial prefrontal cortex. The symbols represent means \pm SEM, and where SEM bars are not evident they are smaller than the diameter of the symbol. Four groups are represented: a saline-treated control group (Saline, $N=12$ ); a group given access to running wheels (Wheel, $N=10$ ); and groups treated with amphetamine (Amph, $N=10$ ) or cocaine (Cocaine, $N=10$ ). Asterisks indicate groups that differed significantly from the control group.



FIG. 3. Effect of treatment on the number of dendritic branches as a function of branch order in groups given access to a running wheel (W), or treated with amphetamine (A) or cocaine $(\mathrm{C})$. Circles indicate that there was a significant decrease (open circles) or a significant increase (closed circles) in the number of dendritic branches at the branch order indicated, relative to the saline-treated control group. A dash indicates that there was no difference from the control. Acc, accumbens; Cg3-A, prefrontal apical dendrites; $\mathrm{Cg} 3-$ $\mathrm{B}$, prefrontal basilar dendrites. 


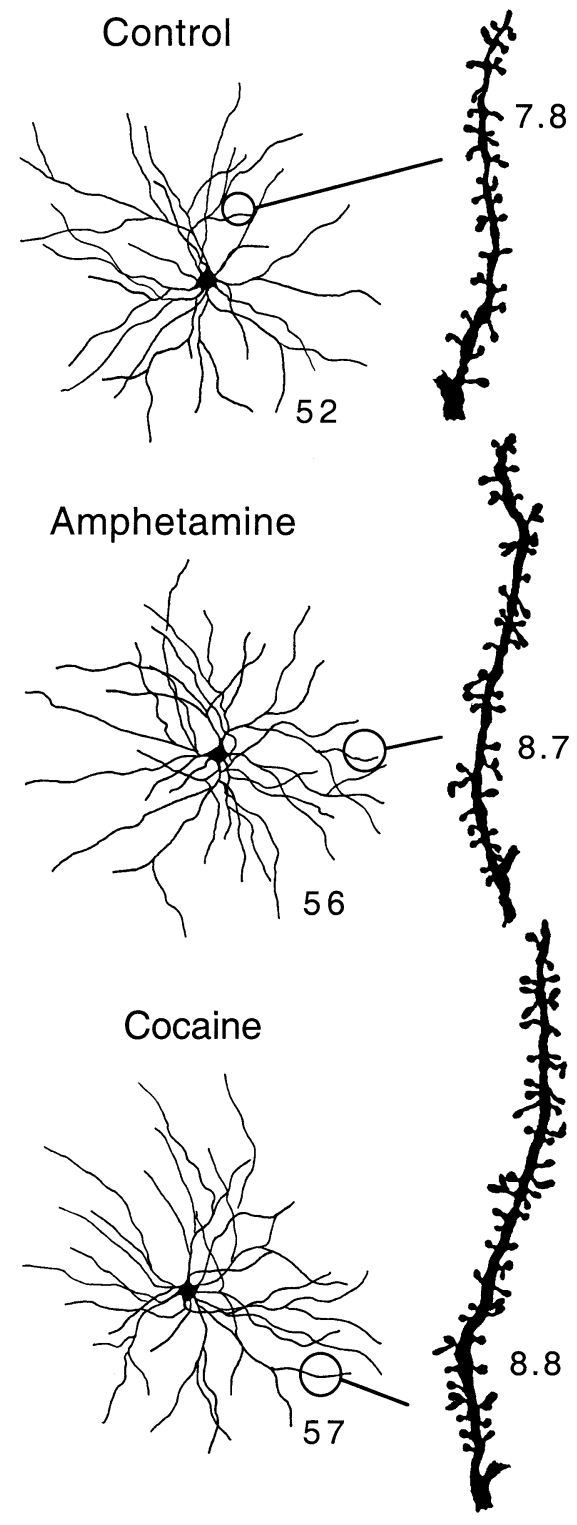

FIG. 4. Camera lucida drawings of representative medium spiny neurons in the shell of the nucleus accumbens from saline- (Control), amphetamine- and cocaine-treated rats. The drawings to the right of each cell represent the dendritic segments (indicated by the pointers) drawn at high power $(1000 \times)$ that were used to calculate spine density. These particular cells were selected for representation because they were very close to the group average. The number at the bottom right of each cell indicates the total number of branches for that cell, and the numbers to the right of each dendritic segment the number of spines $/ 10 \mu \mathrm{m}$ for that dendrite (compare these numbers to the group means given in Fig. 1).

animals, $5.35 \%$ of total spines on the distal dendrites of medium spiny neurons had multiple heads, whereas in amphetamine- and cocaine-treated animals, the percentage of spines with multiple heads approximately doubled (to $14.6 \%$ and $11.0 \%$, respectively, $F=149.0$, $P<0.001)$.

The effects of amphetamine and cocaine on apical dendrites of layer $\mathrm{V}$ pyramidal cells in the cingulate cortex, area 3 (medial prefrontal cortex) $(\mathrm{Cg} 3)$ were very similar to those on medium spiny neurons. Figure $2 \mathrm{~B}$ shows that in amphetamine- and cocaine-treated animals, there was a $26.1 \%$ and $28 \%$ increase, respectively, in the number of $\mathrm{Cg} 3$ apical dendritic branches $(F=26.7, P<0.001)$, with no change in the wheel group. In both drug-treated groups, this effect

\section{Nucleus Accumbens}
A. Total
B. Branched
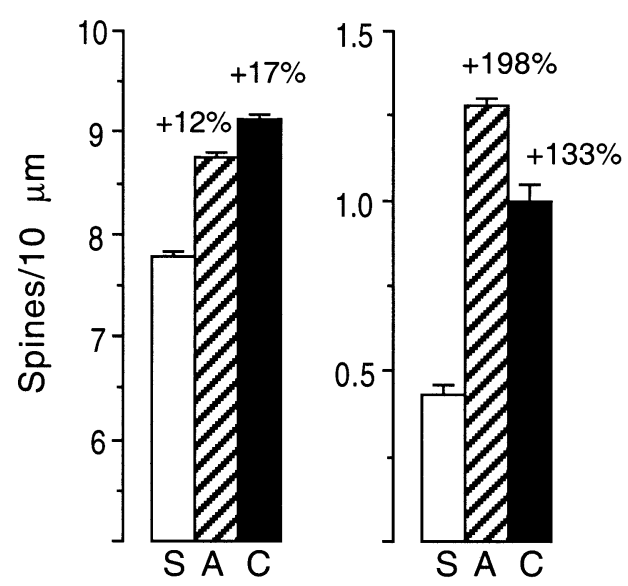

FIG. 5. The mean ( \pm SEM) total number of spines (A) and branched spines (B) on medium spiny neurons in the shell of the nucleus accumbens of saline(S), amphetamine- (A) and cocaine- (C) treated animals. Asterisks indicate groups that differed significantly from the saline-treated control group. The numbers above the bars give the per cent increase in amphetamine- and cocaine-treated animals, relative to the control group.

was confined to relatively proximal dendrites, with statistically significant increases in the number of first-, second-, third- and fourthorder branches, but no significant effect on fifth- or sixth+-order branches (Fig. 3). There was also an increase in the density of spines on $\mathrm{Cg} 3$ apical dendrites in amphetamine- and cocaine-treated animals (by $15.6 \%$ and $23 \%$, respectively), and a decrease in the wheel group $(F=184.0, P<0.001$; Fig. 2E).

The effect of amphetamine on $\mathrm{Cg} 3$ basilar dendrites was very modest, consistent with our previous report (Robinson \& Kolb, 1997). There was no significant effect of amphetamine on the number of $\mathrm{Cg} 3$ basilar branches, and only a $4.2 \%$ increase in spine density (Fig. 2C,F). In contrast, cocaine produced a robust increase in both the number of $\mathrm{Cg} 3$ basilar branches (by $16.5 \%, F=21.45, P<0.001$ ) and the density of spines on $\mathrm{Cg} 3$ basilar dendrites (by $15.9 \%$, $F=75.5, P<0.001$; Fig. 2C,F). In contrast to apical $\mathrm{Cg} 3$ dendrites, the effect of cocaine on the number of basilar branches was confined to only the most distal portion of the dendritic tree (only the effects on fourth-, fifth- and sixth+-order branches were significant; Fig. 3). There was no effect of wheel running experience on $\mathrm{Cg} 3$ basilar branches or spines (Figs $1 \mathrm{~F}$ and 2C).

The analysis of dendritic length using ring intersections yielded a similar pattern of results as the branch order analysis, with the following exceptions (data not shown). First, in the accumbens there was not a statistically significant effect of treatment on the number of ring intersections $(F=2.16)$. Second, for $\mathrm{Cg} 3$ basilar dendrites, the effects of both amphetamine and the wheel experience were statistically significant, although significantly less than the effect of cocaine.

\section{Discussion}

We found that repeated exposure to either amphetamine or cocaine: (i) changed the morphology of neurons in the nucleus accumbens and prefrontal cortex; and (ii) these changes were still evident 1 month (at least 24-25 days) after the discontinuation of drug treatment. Amphetamine and cocaine produced a significant increase in the 
number of dendritic branches, and the density of dendritic spines on medium spiny neurons in the shell of the nucleus accumbens and pyramidal cells in the medial prefrontal cortex. This confirms and extends our earlier report of structural modifications in accumbens and prefrontal cortex neurons following repeated treatment with escalating doses of amphetamine (Robinson \& Kolb, 1997). There are a number of differences between this study and our earlier study that need to be acknowledged. First, in our initial study a very aggressive dosing regimen was used, consisting of escalating doses of amphetamine $(1-10 \mathrm{mg} / \mathrm{kg})$ given twice a day over 5 weeks. In the present study, animals received a more typical 'sensitizing' regimen, consisting of a constant daily dose $(3 \mathrm{mg} / \mathrm{kg}$ of amphetamine or $15 \mathrm{mg} / \mathrm{kg}$ of cocaine) repeated 20 times. Second, males were used in our initial study and females in the present study. Third, the present study included a 'motor activity' control group; and fourth, here the effects of amphetamine were compared with those of cocaine.

This is the first report that both amphetamine and cocaine alter the morphology of neurons in brain regions implicated in drug reward, but in some ways it is not a surprising finding. Other forms of experience-dependent plasticity, e.g. learning (Chang \& Greenough, 1982; Moser et al., 1994; Stewart \& Rusakov, 1995), electrical selfstimulation (Bindu \& Desiraju, 1990; Rao et al., 1993), environmental enrichment (Greenough et al., 1990), recovery of function (Kolb et al., 1998), long-lasting sensitization in Aplysia (Bailey \& Chen, 1983) and mammalian long-term potentiation (Trommald et al., 1990; Moser et al., 1994) are all associated with structural alterations in relevant neural circuits, and other drugs that influence monoamine neurotransmission, e.g. haloperidol (Kerns et al., 1992) and deprenyl (Lakshmana et al., 1998), may alter neuronal morphology. Indeed, it is axiomatic that persistent experience-dependent changes in behaviour are due to alterations in patterns of synaptic connectivity (Hebb, 1949). Given the extremely potent stimulus properties of psychomotor stimulant drugs, and their ability to produce changes in behaviour that last for months to years, it is not surprising that they might also alter synaptic organization.

Of course, we can not tell from the analysis of Golgi-stained material exactly which synapses were modified by amphetamine or cocaine, or in what ways they were rearranged. There is considerable evidence, however, that the kinds of changes in Golgi material described here are indicative of alterations in synaptic organization (Greenough et al., 1990; Kolb et al., 1998). Indeed, dendritic spines are thought to represent the major focus of control over excitatory signalling, and changes in the structure or number of spines the primary means whereby plastic changes can alter synaptic signalling (Horner, 1993; Harris \& Kater, 1994; Rusakov et al., 1996; Shepherd, 1996). For example, an increase in the number of dendritic branches and the number of dendritic spines probably represents an increase in the number of synaptic inputs onto that portion of the dendritic tree, as spines are not usually left vacant (Gray, 1959; Peters \& Feldman, 1976; Wilson et al., 1983). Furthermore, changes in the shape of spines can modify synaptic efficacy by modulating the electrotonic characteristics of the synapse, or the local chemical environment (Horner, 1993; Koch \& Zador, 1993; Shepherd, 1996).

There was an especially intriguing change in the form of dendritric spines on medium spiny neurons following treatment with amphetamine or cocaine. Drug treatment doubled the proportion of spines with multiple heads, i.e. the number of branched spines, consistent with our earlier report (Robinson \& Kolb, 1997). In most brain regions, branched spines are quite rare (Sorra et al., 1998), but there is increasing evidence that they are associated with synaptic plasticity. Trommald et al. $(1996,1990)$ have reported that after longterm potentiation (LTP) evoked by stimulation of the perforant path there is an increase in the number of branched spines on dendrites of dentate granule cells, and Geinisman et al. (1989) found a similar effect after kindling induced by perforant path stimulation. Comery et al. (1996) reported recently that there is an increase in branched spines on medium spiny neurons in the caudate nucleus of rats raised in a complex environment.

Although little is known about branched spines (Harris \& Kater, 1994), recent evidence suggests they may represent a more radical alteration in synaptic organization than might seem at first glance. One hypothesis is that branched spines are formed simply by 'splitting' an existing presynaptic bouton to form two new synapses (Sorra et al., 1998). But, in an evaluation of the largest sample of reconstructed branched spines ever made (from serial EM sections in CA1 of the hippocampus), Sorra et al. (1998) found that of 91 branched spines, 'different branches of the same spine never synapsed with the same presynaptic bouton', and that the boutons on each branch head, 'were not even neighbouring boutons splitting along the same axon' (p. 236). Similarly, in their LTP material, Trommald et al. (1996) reported that 'each of the branches ... made contact with a standard perforant path bouton and showed all the normal attributes of an asymmetric excitatory spine synapse' (p. 224), and, 'after reconstruction of 27 bifurcating spines no case was found in which the same two spine heads were served by the same axon' (p. 225). We do not know if the same holds true for the branched spines found here, but if it does they presumably represent a fundamental reorganization of synaptic inputs onto the distal dendrites of medium spiny neurons in the nucleus accumbens as a consequence of past drug experience.

At the level of analysis used here, the effects of amphetamine and cocaine on medium spiny neurons appeared to be very similar. Both drugs increased dendritic branching, spine density and the number of branched spines to a comparable degree. Furthermore, for both drugs, the effect on the number of dendritic branches was confined to the most distal portion of the dendritic tree. In contrast, in animals allowed access to a running wheel there was not only a decrease (rather than an increase) in dendritic branching, but this was localized to a different portion of the dendritic tree (branch orders 2 and 3). This suggests, of course, that wheel running experience influences different synaptic inputs to psychostimulant drugs, and that the effect of amphetamine and cocaine is not just secondary to their ability to increase motor activity.

It is interesting that both amphetamine and cocaine influenced dendritic branching only on the distal portion of the dendritic tree of medium spiny neurons, because this is thought to be the site of convergence of dopamine and glutamate inputs on these cells (Smith \& Bolam, 1990). Dopamine inputs from the ventral tegmental area and substantia nigra form symmetric contacts with spine necks, or nearby on the dendritic shaft, and excitatory amino acid inputs (presumably glutamate) form asymmetric contacts with spine heads (Totterdell \& Smith, 1989; Sesack \& Pickel, 1990; Smith \& Bolam, 1990; Groenewegen et al., 1991). Similar dopamine/glutamate 'triads' are found on pyramidal cells in the cortex (Goldman-Rakic et al., 1989, 1992; Berger et al., 1991). It is usually assumed that the glutamate inputs to the striatum arise from cells in the cortex, hippocampus or amygdala, but it has been suggested that dopamine neurons themselves may make both symmetric contacts, where dopamine is released, and asymmetric contacts, where the same cell releases glutamate (Hattori, 1993; Sulzer et al., 1998). Whatever the case, these dopamine/glutamate 'triads' are thought to provide the structural means by which dopamine modulates the excitatory drive on neurons (Smith \& Bolam, 1990; Groenewegen et al., 1991; Goldman-Rakic et al., 1992).

Of course, glutamate receptors have been implicated in many 
different forms of plasticity, including the behavioural plasticity associated with repeated exposure to drugs of abuse (Wolf, 1998). This raises the possibility that exposure to amphetamine or cocaine provokes a reorganization of dopamine and glutamate inputs onto nucleus accumbens and prefrontal cortex cells in a way that renders animals hypersensitive, or 'sensitized' to stimuli that engage this circuitry (Pierce \& Kalivas, 1997). The reorganization of dopamine and glutamates inputs could take many different forms (see fig. 5 in Robinson \& Kolb, 1997), and to determine how they are actually rearranged will require detailed ultrastructural studies. Nevertheless, the idea that dopamine inputs may be altered by exposure to psychostimulant drugs is supported by the observation that chronic cocaine alters the levels of neurofilament proteins in the ventral tegmental area (Beitner-Johnson et al., 1992), the origin of dopamine projections to the nucleus accumbens and prefrontal cortex. These neurofilament proteins are components of the neuronal cytoskeleton and could play a role in modifying neuronal morphology (BeitnerJohnson et al., 1992). It is also relevant, therefore, that behavioural sensitization to amphetamine and cocaine is prevented by co-treatment with protein synthesis inhibitors (Robinson, 1991; Karler et al., 1993).

Although the influence of amphetamine and cocaine on the structure of medium spiny neurons appeared to be similar, they had different effects in the prefrontal cortex. Amphetamine had very little effect on the basilar dendrites of prefrontal cortex pyramidal cells. This is in agreement with our earlier study (Robinson \& Kolb, 1997), where we found that amphetamine had no significant effect on the basilar dendrites of $\mathrm{Cg} 3$ pyramidal cells. [The small effect seen here could be because in the present study we used female rather than male rats, and females show more robust sensitization (Robinson, 1984), or, because in the present study we sampled layer $\mathrm{V}$ rather than layer III pyramids]. Cocaine, on the other hand, produced a robust increase in both the number of basilar dendritic branches on $\mathrm{Cg} 3$ pyramidal cells and on spine density. This suggests, therefore, that cocaine alters synaptic inputs onto both apical and basilar dendrites of $\mathrm{Cg} 3$ pyramids, whereas the effect of amphetamine is confined primarily to the apical dendrites.

It is impossible to know from the present data what inputs to the prefrontal cortex might be differentially affected by cocaine versus amphetamine, as very little is known about the spatial arrangement of neurochemically specific synapses onto $\mathrm{Cg} 3$ pyramidal cells. Nevertheless, the distribution of neurotransmitters and neurotransmitter receptors in the cortex is highly laminar, consistent with the notion that the apical and basilar dendrites receive neurochemically distinct inputs (Zilles et al., 1990). Interestingly, the dopamine innervation of these cells may be an exception to this rule. Krimer et al. (1997) reported recently that in the prefrontal cortex of macaque monkeys, tyrosine hydroxylase-positive (presumably dopamine) appositions were evenly distributed over the entire dendritic tree of pyramidal cells. On the other hand, 5- $\mathrm{HT}_{1}$-type receptors are highly concentrated in layer $\mathrm{V}$, in the region of the basilar dendrites (Zilles et al., 1990). Although highly speculative, it is possible that the differential influence of cocaine versus amphetamine on the basilar dendrites of pyramidal cells is related to the fact that cocaine has a greater influence on serotonin neurotransmission than does amphetamine (see White et al., 1998 for references). Whatever the case, these data suggest that even closely related drugs of abuse, e.g. cocaine and amphetamine, may produce different patterns of synaptic reorganization. This is consistent with accumulating evidence that different mechanisms are responsible for the sensitization produced by repeated treatment with cocaine versus amphetamine (White et al., 1998). Unravelling how different patterns of synaptic reorganization contribute to the function of a given neural circuit, and thus to a particular behavioural outcome, poses a major challenge for future research.

In closing, the molecular mechanisms by which amphetamine or cocaine might produce the kinds of changes in neuronal structure described here is not known, but a number of possibilities have been identified. Amphetamine and cocaine induce a variety of immediate early genes in both the cortex and striatum (see Graybiel et al., 1990; Harlan \& Garcia, 1998 for review). These regulate the transcription of other target genes, some of which could promote structural adaptations. In addition, both amphetamine and cocaine increase the expression of 'arc' (activity-regulated cytoskeleton), an 'effector immediate early gene' that encodes for a protein found in neuronal cell bodies and dendrites, and is thought to be a component of the cytoskeleton (Fosnaugh et al., 1995; Kodama et al., 1998). Furthermore, amphetamine increases arc mRNA in striatal medium spiny neurons and the frontal cortex, and the ability of psychostimulants to induce arc is attenuated by dopamine D1 receptor antagonists, MK-801 or 6-hydroxydopamine (6-OHDA) lesions (Fosnaugh et al., 1995; Kodama et al., 1998). Arc provides, therefore, at least one potential mechanism by which exposure to amphetamine or cocaine, via their ability to increase the extracellular concentrations of monoamines, could trigger a cascade of molecular events leading to structural modifications in neurons that receive monoaminergic inputs.

\section{Acknowledgements}

We gratefully acknowledge G. Gorney, R. Gibb and J. Aerni for excellent technical assistance. This research was supported by grants from the National Institute on Drug Abuse (USA) and the National Science and Engineering Research Council of Canada.

\section{Abbreviations}

6-OHDA, 6-hydroxydopamine; $\mathrm{Cg} 3$, cingulate cortex, area 3 (medial prefrontal cortex); LTP, long-term potentiation.

\section{References}

Bailey, C.H. \& Chen, M. (1983) Morphological basis of long-term habituation and sensitization in Aplysia. Science, 220, 91-93.

Beitner-Johnson, D., Guitart, X. \& Nestler, E.J. (1992) Neurofilament proteins and the mesolimbic dopamine system: common regulation by chronic morphine and chronic cocaine in the rat ventral tegmental area. $J$. Neuroscience, 12, 2165-2176.

Berger, B., Gaspar, P. \& Verney, C. (1991) Dopaminergic innervation of the cerebral cortex: unexpected differences between rodents and primates. Trends Neurosci., 14, 21-27.

Bindu, P.N. \& Desiraju, T. (1990) Increase of dendritic branching of CA3 neurons of hippocampus and self-stimulation areas in subjects experiencing self-stimulation of lateral hypothalamus and substantia nigra-ventral tegmental area. Brain Res., 527, 171-175.

Camp, D.M. \& Robinson, T.E. (1988) Susceptibility to sensitization. II. The influence of gonadal hormones on enduring changes in brain monoamines and behavior produced by the repeated administration of D-amphetamine or restraint stress. Behav. Brain Res., 30, 69-88.

Chang, F.L. \& Greenough, W.T. (1982) Lateralized effects of monocular training on dendritic branching in adult split-brain rats. Brain Res., 232, 283-292.

Coleman, P.D. \& Riesen, A.H. (1968) Environmental effects on cortical dendritic fields. I. Rearing in the dark. J. Anat., 102, 363-374.

Comery, T.A., Stamoudis, C.X., Irwin, S.A. \& Greenough, W.T. (1996) Increased density of multiple-head dendritic spines on medium-sized spiny neurons of the striatum in rats reared in a complex environment. Neurobiol. Learn. Mem., 66, 93-96.

Davis, W.M. \& Smith, S.G. (1976) Role of conditioned reinforcers in the initiation, maintenance and extinction of drug-seeking behavior. Pavlov J. Biol. Sci., 11, 222-236. 
Fosnaugh, J.S., Bhat, R.V., Yamagata, K., Worley, P.F. \& Baraban, J.M. (1995) Activation of arc, a putative 'effector' immediate early gene, by cocaine in rat brain. J. Neurochem., 64, 2377-2380.

Geinisman, Y., Morrell, F. \& de Toledo-Morrell, L. (1989) Perforated synapses on double-headed dendritic spines: a possible structural substrate of synaptic plasticity. Brain Res., 480, 326-329.

Gibb, R. \& Kolb, B. (1998) A method for vibratome sectioning of Golgi-Cox stained whole rat brain. J. Neurosci. Meth., 79, 1-4.

Goldman-Rakic, P.S., Leranth, C., Williams, M.S., Mons, N. \& Geffard, M. (1989) Dopamine synaptic complex with pyramidal neurons in primate cerebral cortex. Proc. Natl. Acad. Sci. USA, 86, 9015-9019.

Goldman-Rakic, P.S., Lidow, M.S., Smiley, J.F. \& Williams, M.S. (1992) The anatomy of dopamine in monkey and human prefrontal cortex. J. Neural Transm., 36, 163-177.

Gray, E.G. (1959) Axo-somatic and axo-dendritic synapses of the cerebral cortex: an electron microscope study. J. Anat., 93, 420-433.

Graybiel, A.M., Moratalla, R. \& Robertson, H.A. (1990) Amphetamine and cocaine induce drug-specific activation of the c-fos gene in striosomematrix compartments and limbic subdivisions of the striatum. Proc. Natl. Acad. Sci. USA, 87, 6912-6916.

Greenough, W.T., Withers, G.S. \& Wallace, C.S. (1990) Morphological changes in the nervous system arising from behavioral experience: what is the evidence that they are involved in learning and memory? In Squire, L.R. and Lindenlaub, E. (eds) The Biology of Memory, Symposia Medica Hoechst. F. K. Schattauder, New York, pp. 159-185.

Groenewegen, H.J., Berendse, H.W., Meredith, G.E., Haber, S.N., Voorn, P., Wolters, J.G. \& Lohman, A.H.M. (1991) Functional anatomy of the ventral, limbic system-innervated striatum. In Willner, P. and Scheel-Kruger, J. (eds) The Mesolimbic Dopamine System: From Motivation to Action. John Wiley, New York, pp. 19-59.

Harlan, R.E. \& Garcia, M.M. (1998) Drugs of abuse and immediate-early genes in the forebrain. Mol. Neurobiol., 16, 221-267.

Harris, K.M. \& Kater, S.B. (1994) Dendritic spines: cellular specializations imparting both stability and flexibility to synaptic function. Annu. Rev. Neurosci., 17, 341-371.

Hattori, T. (1993) Conceptual history of the nigrostriatal dopamine system. Neurosci. Res., 16, 239-262.

Hebb, D.O. (1949) The Organization of Behavior. John Wiley, New York.

Horner, C.H. (1993) Plasticity of the dendritic spine. Prog. Neurobiol., 41, 281-321

Kalivas, P.W. \& Stewart, J. (1991) Dopamine transmission in the initiation and expression of drug- and stress-induced sensitization of motor activity. Brain Res. Rev., 16, 223-244.

Karler, R., Finnegan, K.T. \& Calder, L.D. (1993) Blockade of behavioral sensitization to cocaine and amphetamine by inhibitors of protein synthesis. Brain Res., 603, 19-24.

Kerns, J.M., Sierens, D.K., Kao, L.C., Klawans, H.L. \& Carvey, P.M. (1992) Synaptic plasticity in the rat striatum following chronic haloperidol treatment. Clin. Neuropharmacol., 15, 488-500.

Koch, C. \& Zador, A. (1993) The function of dendritic spines: devices subserving biochemical rather than electrical compartmentalization. $J$. Neuroscience, 13, 413-422.

Kodama, M., Akiyama, K., Ujike, H., Shimizu, Y., Tanaka, Y. \& Kuroda, S (1998) A robust increase in expression of arc gene, an effector immediate early gene, in the rat brain after acute and chronic methamphetamine administration. Brain Res., 796, 273-283.

Kolb, B., Forgie, M., Gibb, R., Gorny, G. \& Rowntree, S. (1998) Age, experience and the changing brain. Neurosci. Biobehav. Rev., 22, 143-159.

Krimer, L.S., Jakab, R.L. \& Goldman-Rakic, P.S. (1997) Quantitative threedimensional analysis of the catecholaminergic innervation of identified neurons in the macaque prefrontal cortex. J. Neuroscience, 17, 7450-7461.

Kuczenski, R. (1983) Biochemical actions of amphetamine and other stimulants. In Creese, I. (ed.) Stimulants: Neurochemical, Behavioral and Clinical Perspectives. Raven Press, New York, pp. 31-61.

Lakshmana, M.K., Rao, B.S., Dhingra, N.K., Ravikumar, R., Govindaiah, R., Meti, B.L. \& Raju, T.R. (1998) Chronic (-) deprenyl administration increases dendritic arborization in CA3 neurons of hippocampus and $\mathrm{AChE}$ activity in specific regions of the primate brain. Brain Res., 796, 38-44.

Moser, M.B., Trommald, M. \& Andersen, P. (1994) An increase in dendritic spine density on hippocampal CA1 pyramidal cells following spatial learning in adult rats suggests the formation of new synapses. Proc. Natl. Acad. Sci. USA, 91, 12 673-12 675.

Nestler, E.J., Hope, B.T. \& Widnell, K.L. (1993) Drug addiction: a model for the molecular basis of neural plasticity. Neuron, 11, 995-1006.
Peters, A. \& Feldman, M.L. (1976) The projection of the lateral geniculate nucleus to area 17 of the rat cerebral cortex. I. General description. $J$. Neurocytol., 5, 63-84.

Pierce, R.C. \& Kalivas, P.W. (1997) A circuitry model of the expression of behavioral sensitization to amphetamine-like psychostimulants. Brain Res. Rev., 25, 192-216.

Rao, B.S., Desiraju, T. \& Raju, T.R. (1993) Neuronal plasticity induced by self-stimulation rewarding experience in rats - a study on alteration in dendritic branching in pyramidal neurons of hippocampus and motor cortex. Brain Res., 627, 216-224.

Robinson, T.E. (1984) Behavioral sensitization: characterization of enduring changes in rotational behavior produced by intermittent injections of amphetamine in male and female rats. Psychopharmacol., 84, 466-475.

Robinson, T.E. (1991) The neurobiology of amphetamine psychosis: evidence from studies with an animal model. In Nakazawa, T. (ed.) Taniguchi Symposia on Brain Sciences, Vol. 14, Biological Basis of Schizophrenia. Japan Scientific Societies Press, Tokyo, pp. 185-201.

Robinson, T.E. \& Becker, J.B. (1986) Enduring changes in brain and behavior produced by chronic amphetamine administration: a review and evaluation of animal models of amphetamine psychosis. Brain Res. Rev., 11, 157-198.

Robinson, T.E. \& Berridge, K.C. (1993) The neural basis of drug craving: An incentive-sensitization theory of addiction. Brain Res. Rev., 18, 247-291.

Robinson, T.E. \& Kolb, B. (1997) Persistent structural modifications in nucleus accumbens and prefrontal cortex neurons produced by previous experience with amphetamine. J. Neuroscience, 17, 8491-8497.

Rusakov, D.A., Stewart, M.G. \& Korogod, S.M. (1996) Branching of active dendritic spines as a mechanism for controlling synaptic efficacy. Neuroscience, 75, 315-323.

Sesack, S.R. \& Pickel, V.M. (1990) In the rat medial nucleus accumbens, hippocampal and catecholaminergic terminals converge on spiny neurons and are in apposition to each other. Brain Res., 527, 266-279.

Shepherd, G.M. (1996) The dendritic spine: a multifunctional integrative unit. J. Neurophysiol., 75, 2197-2210.

Sholl, D.A. (1981) The Organization of the Cerebral Cortex. Methuen, London.

Smith, A.D. \& Bolam, J.P. (1990) The neural network of the basal ganglia as revealed by the study of synaptic connections of identified neurons. Trends Neurosci., 13, 259-265.

Sorra, K.E., Fiala, J.C. \& Harris, K.M. (1998) Critical assessment of the involvement of perforations, spinules, and spine branching in hippocampal synapse formation. J. Comp. Neurol., 398, 225-240.

Stewart, J. \& Badiani, A. (1993) Tolerance and sensitization to the behavioral effects of drugs. Behav. Pharmacol., 4, 289-312.

Stewart, M.G. \& Rusakov, D.A. (1995) Morphological changes associated with stages of memory formation in the chick following passive avoidance training. Behav. Brain Res., 66, 21-28.

Sulzer, D., Joyce, M.P., Lin, L., Geldwert, D., Haber, S.N., Hattori, T. \& Rayport, S. (1998) Dopamine neurons make glutamatergic synapses in vitro. J Neuroscience, 18, 4588-4602.

Tomie, A. (1996) Locating reward cue at response manipulandum (CAM) induces symptoms of drug abuse. Neurosci. Biobehav. Rev., 20, 505-535.

Totterdell, S. \& Smith, A.D. (1989) Convergence of hippocampal and dopaminergic input onto identified neurons in the nucleus accumbens of the rat. J. Chem. Neuroanat., 2, 285-298.

Trommald, M., Hulleberg, G. \& Andersen, P. (1996) Long-term potentiation is associated with new excitatory spine synapses on rat dentate granule cells. Learning \& Memory, 3, 218-228.

Trommald, M., Vaaland, J.L., Blackstad, T.W. \& Andersen, P. (1990) Dendritic spine changes in rat dentate granule cells associated with long-term potentiation. In Guidotti, A. (ed.) Neurotoxicity of Excitatory Amino Acids. Raven Press, New York, pp. 163-174.

White, F.J., Amit, J., Koeltzow, T.E. \& Hu, X.-T. (1998) Dopamine receptor antagonists fail to prevent induction of cocaine sensitization. Neuropsychopharmacol., 18, 26-40.

White, F.J. \& Wolf, M.E. (1991) Psychomotor stimulants. In Pratt, J. (ed.) The Biological Bases of Drug Tolerance and Dependence. Academic Press, New York, pp. 153-197.

Wilson, C.J., Groves, P.M., Kitai, S.T. \& Linder, J.C. (1983) Three-dimensional structure of dendritic spines in the rat neostriatum. J. Neuroscience, $\mathbf{3}$, 383-398.

Wolf, M.E. (1998) The role of excitatory amino acids in behavioral sensitization to psychomotor stimulants. Prog. Neurobiol., 54, 679-720.

Zilles, K. (1985) The Cortex of the Rat. Springer, Berlin.

Zilles, K., Wree, A. \& Dausch, N.-D. (1990) Anatomy of the neocortex: neurochemical organization. In Kolb, B. and Tees, R.C. (eds) The Cerebral Cortex of the Rat. MIT Press, Cambridge, MA, USA, pp. 113-150. 